J run things at this

throughput that helped us realize where the next bottleneck would be, which is turning these hits into lead compounds," says Anson.

\section{Into the cell}

The vast majority of compounds identified as hits in screening fail in the later stages of lead development, usually because they are biologically unsuitable for development as a medicine. It makes sense to find out more about the biological workings of a hit compound as early as possible, before you spend time and money on further development.

"One area where we are seeing continuing demand for products in the early stages of drug discovery involves cell-based assays," says Marc Feiglin, biopharma technology officer at life-sciences equipment supplier Tecan, based in Männedorf, Switzerland. Tecan's Cellerity system provides an automated system for high-throughput cellculture production and assay preparation.

"One of the reasons people are using cell-based assays is they're starting to get earlier indications of problems that may occur later on," says Anson. GE Healthcare's IN Cell Analyzer platforms offer real-time high-resolution cellular imaging, allowing researchers to construct high-throughput intracellular assays that were previously impossible (see 'Proteins on the move', below).
Cell-based assays produce much more information on the effects of a potential drug compound than in vitro assays can, and this enables researchers to get more useful data as early in the discovery process as possible, says Mark Collins, senior product manager at Cellomics, a cell-assay platform developer based in Pittsburgh, Pennsylvania. "You can screen millions of compounds very quickly, but the challenge for pharma is getting enough information out of that screening to make considered decisions to move forwards," he says.

Cellomics' high-content screening platforms such as the ArrayScan and KineticScan readers use fluorescence probes to obtain richer measurements of cellular activity. "The point is that you're measuring more than one thing that's going on, and analysing images in an automated fashion to get quantitative data," Collins says. "You can address biology you could never have understood before, things like morphology screening that you just can't do if you've smashed the cell up and put it in a test tube."

\section{Getting the knowledge}

With both throughputs and the richness of assay measurements increasing, making sense of the vast amounts of data generated can be a bottleneck. Informatics or knowl- edge-management systems are a necessity, and many platform providers offer integrated software packages.

"When you are generating multiple images and need to get a quantitative image out quickly, you're not going to do that by running millions of spreadsheets or looking at millions of images," says Collins. "What our informatics package does is automate that whole process of linking data together and linking images with numbers and storing that in a very robust database. The image is always there as a final answer, but once the instrument has done its job you're looking at numbers, which is a sight easier than looking at 1,000 images."

Informatics can also help reduce attrition by improving the selection of hits to take into further development. "By integrating our synthetic chemistry work with a knowledgemanagement approach, we've demonstrated that we can improve the interaction profile of a customer's project by increasing the number of lead series they have for each target, which gives them more shots at the goal," says Ed Hodgkin, vice-president for business development at discovery services group Tripos, based in St Louis, Missouri.

In a collaboration with New York-based pharmaceutical giant Pfizer, Tripos has developed the SARNavigator suite of tools for analysing high-throughput screening results. The package allows researchers to examine structure-activity relations (SAR) quantitatively to help determine which hits should go forward into the lead identification and optimization phase. "That combination of SARNavigator and the chemical compounds we

\title{
PROTEINS ON THE MOVE
}

Intracellular signalling pathways are involved in virtually every disease process, and cell-surface

receptor proteins that initiate these pathways are favoured drug targets (see Nature 428, 225-233; 2004). In the intracellular pathways leading from these receptors, signalling proteins move within the cell - from the cytoplasm into the nucleus, for example. Testing the effects of potential drug compounds against these intracellular protein movements has, unsurprisingly, proved much harder than testing against proteins at the surface of the cell. The ability to do so promises to find drugs with new modes of action and greater selectivity.

A leader in this field is Biolmage in Copenhagen, Denmark, which focuses on green fluorescent proteins (GFP) as a tool in drug discovery. Biolmage's proprietary Redistribution technology uses genetically encoded GFP tags to track protein translocation in living cells, and so identify compounds that inhibit onward transmission of the signal. "It's pretty clear now that every signalling pathway has components that must move in the cell for the signal interaction to occur," says Len Pagliaro, vicepresident for business development at Biolmage. "If you take these targets and

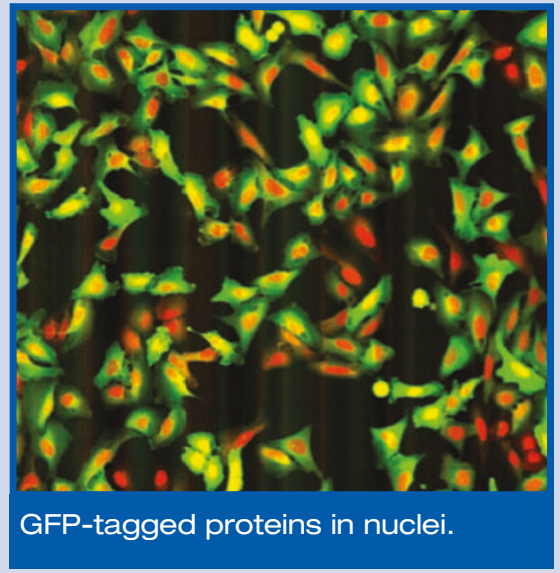

track them, you can measure movement in response to potential stimulators and agonists, and have the potential to find compounds that inhibit that movement."

The firm deploys GE Healthcare's IN Cell Analyzer to carry out high-throughput imaging for screening and analysis of the effects of members of its own library of over 250,000 compounds. Such intracellular assays provide the opportunity to discover drugs that interact with target proteins in new ways - by targeting protein localization domains rather than catalytic domains, for instance. Studying living cells also creates more opportunities for serendipitous discoveries.

"Obviously, cell-based assays are inherently a somewhat dirty system biologically, and that has both benefits and liabilities," Pagliaro notes. "The benefit is that in principle it's more like the pathway you're going to see in vivo. The liability is that when you get a hit, you don't know that the compound is acting on the target that you want to see it acting on. We like that aspect, because that can deliver very interesting progressible hits that we would not have found otherwise." 